\title{
FEATURES OF CREATING OF WORKPIECES MODELS BY MEANS OF VISION SYSTEMS
}

\author{
Vladimir Filaretov, Dmitry Yukhimets, Alexander Zuev, Anton Gubankov, \\ Eduard Mursalimov, Igor Gornostaev
}

Institute of Automation and Control Processes FEB RAS, 5 Radio St., Vladivostok 690041, Russia Far Eastern Federal University, 8 Suhanova St., Vladivostok 690950, Russia

\begin{abstract}
In the paper an algorithm of determining the gaps in the point clouds obtained after scanning of the processed workpieces by means of vision systems is proposed. The resulting point cloud is projected on a plane, the location of which is set in advance for each type of workpieces. Next, the resulting area of projection is estimated, visible points are allocated and their density on the obtained part of model is determined. In this analysis of the quality of point clouds are proposed to use of relatively simple, well-known and proven algorithms for processing of raster images. The efficiency and effectiveness of this algorithm is confirmed by a model example.
\end{abstract}

Keyword: vision system; point cloud; scanning; image processing; CAD-model; workpiece
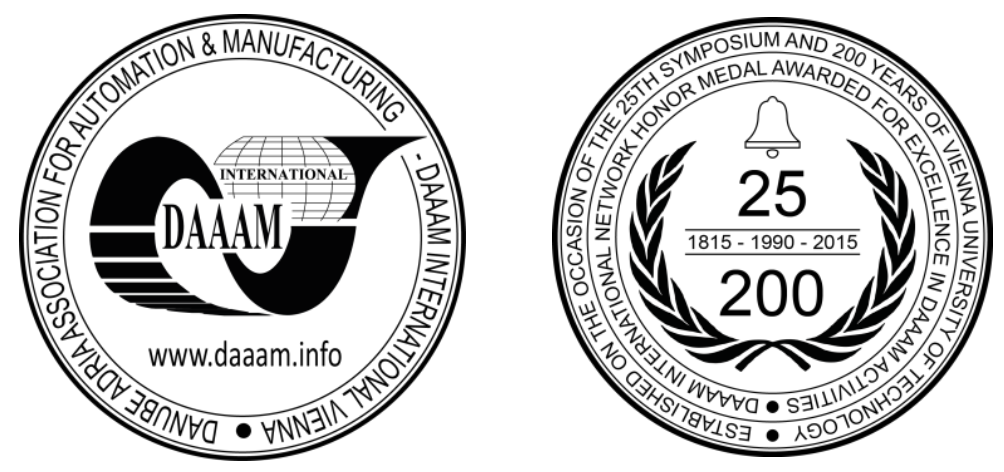

This Publication has to be referred as: Filaretov, V[ladimir]; Yukhimets, D[mitry]; Zuev, A[lexander]; Gubankov, A[nton]; Mursalimov, E[duard] \& Gornostaev, I[gor] (2016). Features of Creating of Workpieces Models by Means of Vision Systems, Proceedings of the 26th DAAAM International Symposium, pp.0292-0300, B. Katalinic (Ed.), Published by DAAAM International, ISBN 978-3-902734-07-5, ISSN 1726-9679, Vienna, Austria DOI:10.2507/26th.daaam.proceedings.040 


\section{Introduction}

One of the perspective directions of using of sensing industrial robots (SIR) in the industry is the automation of various technological operations (assembling, milling, cutting, etc.) when the position and orientation of workpieces in working area of SIR are previously unknown. Creation of the algorithms for fast and accurate definition of position and orientation of the working object in the base coordinate system of SIR is a very important task.

Research showed that to solve this problem it is reasonable to use modern vision systems (VS) [1-8] allowing to obtain precise data of position and orientation of various spatial details. As was defined as VS for creation of threedimensional models of workpieces it is good to use optical and laser scanners and for identification of the changing working environment in real-time scale - stereo cameras.

The workpieces models created by means of scanners represents clouds of points in three-dimensional space. One of the features of creation of three-dimensional models by means of the VS is that a scanner (or a camera) has limited visibility area and for one scanning period it is possible to receive only limited part of the workpiece model which may contain together with noise also gaps. And this does not allow to create full model at the stationary position of the VS or workpiece. Thus the combination of the clouds of points, received on every scanning period to the single cloud describing the whole model, is the main objective, which needs to be solved when developing algorithms of creation of the workpieces models by means of the VS. However, the obtained cloud of points in addition to noise may contain gaps, the presence of which will not allow getting suitable CAD model of workpiece or will make a significant error in determining of its position and orientation. Therefore it is very important to estimate quality of the combined cloud before using, that is to determine the presence and size of gaps, and if necessary to rescan the necessary part.

As a result, in this paper the problem of developing of an algorithm for determining the gaps in the clouds of points that obtained after scanning is set and solved. In addition, there is a validation of the proposed algorithm on a model example.

\section{Developing of algorithm of gaps definition in the workpieces models}

One of the main functions of the information-control systems of SIR is creating of the precise models of environment, which are used at formation of the movement trajectories for the tool center point (TCP). Thus, the main information sources about working environment are VS and sensors, defining the position of TCP and the service equipment (positioners, fixing system for details, etc.).

Despite of type of VS creation of workpieces models is going on the generalized algorithm, the flow graph is showed on figure 1 . The figure shows that the considered algorithm consists on the following steps.

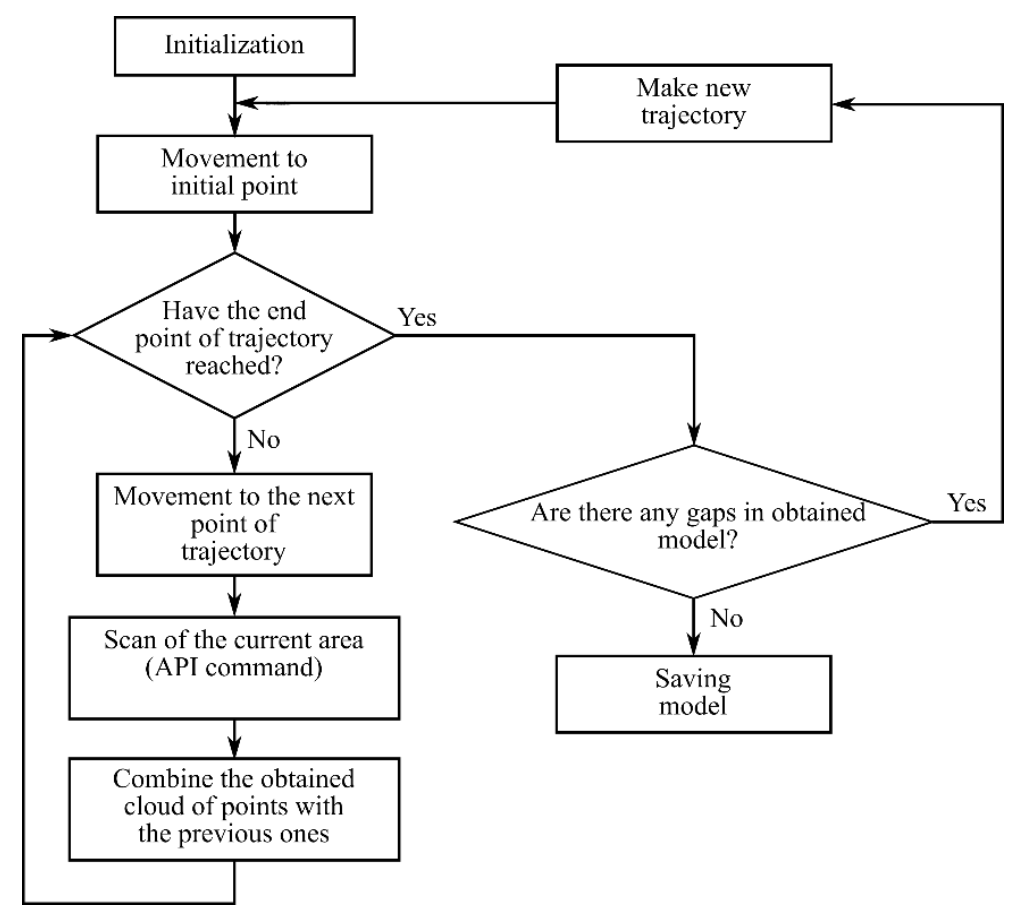

Fig. 1. Flow graph of generalized algorithm of creating of workpieces models

1) Initialization. On this step the workpiece type is selected from database and parameters of scanning process for chosen workpiece (VS initial position or positioning devices, movement trajectory of VS or workpieces, movement mode and so on) are loaded. The equipment involved in scanning process (VS, movement system) is initialized. If there 
are mistakes during the initialization process, then the corresponding messages are sent to diagnostic subsystem of information control system and scanning process stops till debugging.

2) Moving of VS (or workpiece) to initial position for scanning. On this step VS moves to initial position depending on the chosen workpiece type. Then the first scanning step is carried out which sets the position of the coordinate frame for model.

3) Movement of VS (or workpiece) on the set step in linear and/or angular coordinates. The direction and value of this movement are set by trajectory of scanning which is defined individually for each type of the workpiece.

4) Obtaining a cloud of points for the current position of the VS (or workpiece). This operation is carried out on a call of the corresponding commands of the built-in software of VS. The result of command execution is:

- for optical scanner or stereo camera - cloud of points describing part of working area;

- for the laser scanner - cloud of points describing profile of surface of workpiece, belonging to the scanning plane.

It should be noted that scanning process has to be synchronized with movement of the VS or workpiece. For the laser scanner this synchronization can be carried out at the hardware level (the scanner has entrances for position sensors of the device of scanner movement). For optical scanner only program synchronization is possible.

5) Reduction of the received cloud of points to base coordinate system (CS) and combination with the previous cloud of points. This operation depends on that moves during the scanning: VS or workpiece.

6) Repetition of steps 3 - 5 until the whole set trajectory of scanning will be passed.

7) Checking of the created model for gaps. In the created three-dimensional model of workpiece can be gaps parts of workpiece model where VS cannot compare points of three-dimensional model to the scanned surface (see fig. 2). It depends on properties of the scanned surface.

Existence of a large number of gaps specifies that the created model gives poor information and cannot be suitable for further using for formation of the movement trajectory of TCP of SIR. For an exception of such situations, the quality of the created model is checked on this algorithm step. A quality indicator of the model is the average density $\rho$ of the cloud of points that is number of points per unit of model surface. If this density is less than set value, then the created model has gaps and scanning of certain parts is necessary to repeat.

In a general view algorithm of definition of gaps in the workpieces models is as follows. The received cloud of points is projected on the plane, which position is set in advance for each workpiece type. This procedure is briefly shown in figure 3.

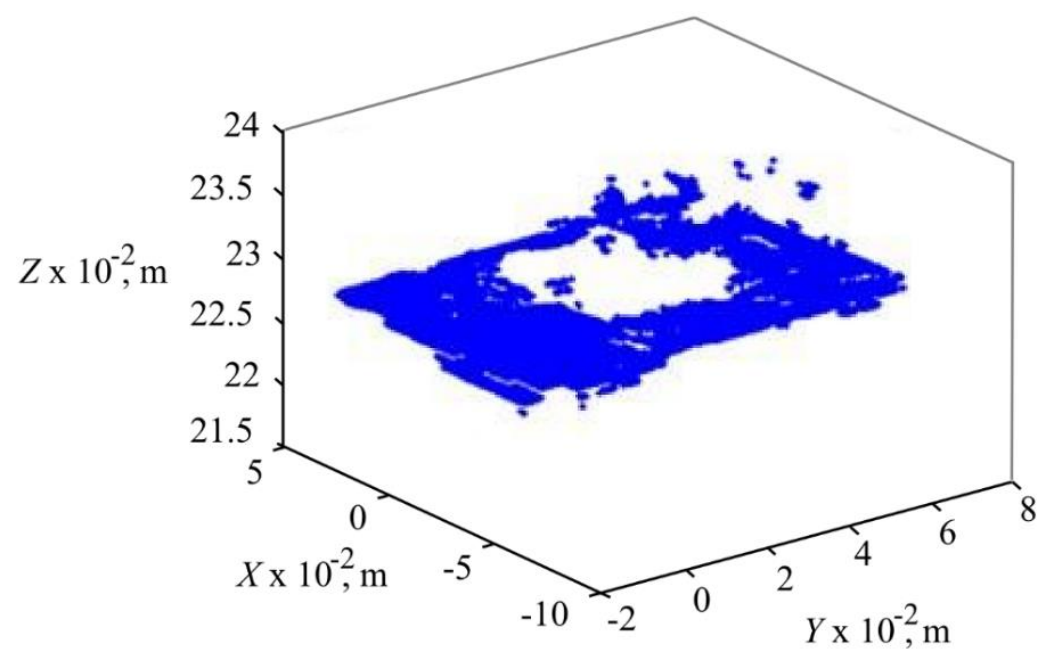

Fig. 2. $\neg$ An example of cloud of points with a gap

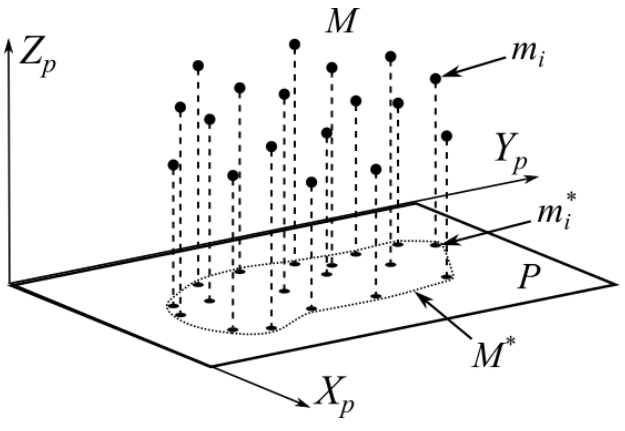

Fig. 3. Projection of received cloud of points to the set plane

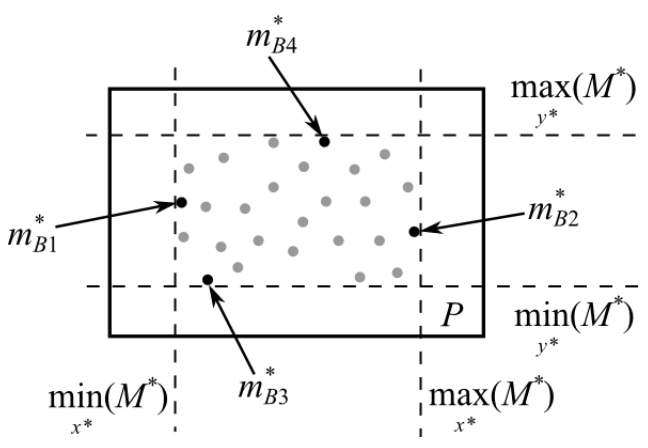

Fig. 4. The estimation of projection area of cloud $M^{*}$ to the plane $\mathrm{P}$ 
The initial cloud of points $M=\left\{m_{i}\right\}, i=\overline{1, N}$ defined in the base CS transformed into the cloud of points $M^{*}=\left\{m_{i}^{*}\right\}, i=\overline{1, N}$ defined in CS $X_{p} Y_{p} Z_{p}$ connected with the plane $P$ on which projection is carried out. The origin of this CS coincides with the origin of base CS, and axes $X_{p}$ and $Y_{p}$ belong to this plane. Recalculation of points coordinates of the initial cloud $M$ in coordinates points of the cloud $M^{*}$ is carried out by means of the following transformation:

$$
m_{i}^{*}=R_{p}^{*} m_{i}, i=\overline{1, N}
$$

where $m_{i}=\left(x_{i}, y_{i}, z_{i}\right), m_{i}^{*}=\left(x_{i}^{*}, y_{i}^{*}, z_{i}^{*}\right)$ are respectively coordinates of points of initial $M$ and transformed $M^{*}$ clouds of points; $R_{p}^{*} \in R^{3 \times 3}$ is transition matrix from base CS to $X_{p} Y_{p} Z_{p}$.

After that, one can define the area, which is formed by projections of points $m_{i}^{*}$ to the plane $P$. Since workpieces can have a difficult form then the area of projection can be estimated as follows (see figure 4).

Points with following coordinates are selected from the cloud of points $M^{*}$ :

$$
m_{B 1}^{*}=\min _{x^{*}}\left(M^{*}\right), m_{B 2}^{*}=\max _{x^{*}}\left(M^{*}\right), m_{B 3}^{*}=\min _{y^{*}}\left(M^{*}\right), m_{B 4}^{*}=\max _{y^{*}}\left(M^{*}\right)
$$

These points set a rectangle in which the projection of a cloud $M^{*}$ on the plane $P$ is inscribed. Next, we exclude points, which correspond to the invisible for projection side of workpiece. Thus in the beginning the minimum $z_{\min }^{*}$ and maximum $z_{\max }^{*}$ values of coordinate $z^{*}$ in the cloud of points $M^{*}$ are defined. If these values have different signs, then it means that the plane $P$ divides the cloud of points on two parts. To simplify further analysis it is necessary to have the cloud of points $M^{*}$ on one side of the plane $P$. For this, it is necessary to carry out the following steps:

$$
\tilde{m}_{i}^{*}=m_{i}^{*}-\left(0,0, z_{\min }^{*}\right)^{T}, i=\overline{1, N}
$$

As a result the cloud of points $M^{*}$ will be transformed to the cloud $\tilde{M}^{*}$, which lower point $\tilde{m}_{\min Z}^{*}$ belongs to the plane $P$. After calculating of points $\tilde{m}_{B j}^{*}=\left(\tilde{x}_{B j}^{*}, \tilde{y}_{B j}^{*}, \tilde{z}_{B j}^{*}\right), j=\overline{1,4}$ through them will pass two planes $S_{1}$ and $S_{2}$, which will slit the cloud $\tilde{M}^{*}$ on two parts: part of workpiece surface projected on the plane $P$, and part that is invisible for the projection. These planes are shown in figure 5 and defined as follows.

1) If the maximum value of coordinate $\tilde{z}_{B j}^{*}$ corresponds to points $\tilde{m}_{B 1}^{*}$ or $\tilde{m}_{B 2}^{*}$, then planes $S_{1}$ and $S_{2}$ are defined according to equations [9]:

$$
S_{1}\left(\tilde{x}^{*}, \tilde{y}^{*}, \tilde{z}^{*}\right)=\left|\begin{array}{ccc}
\tilde{y}_{B 1}^{*} & \tilde{z}_{B 1}^{*} & 1 \\
\tilde{y}_{B 3}^{*} & \tilde{z}_{B 3}^{*} & 1 \\
\tilde{y}_{B 4}^{*} & \tilde{z}_{B 4}^{*} & 1
\end{array}\right| \tilde{x}^{*}+\left|\begin{array}{ccc}
\tilde{z}_{B 1}^{*} & \tilde{x}_{B 1}^{*} & 1 \\
\tilde{z}_{B 3}^{*} & \tilde{x}_{B 3}^{*} & 1 \\
\tilde{z}_{B 4}^{*} & \tilde{x}_{B 4}^{*} & 1
\end{array}\right| \tilde{y}^{*}+\left|\begin{array}{ccc}
\tilde{x}_{B 1}^{*} & \tilde{y}_{B 1}^{*} & 1 \\
\tilde{x}_{B 3}^{*} & \tilde{y}_{B 3}^{*} & 1 \\
\tilde{x}_{B 4}^{*} & \tilde{y}_{B 4}^{*} & 1
\end{array}\right| \tilde{z}^{*}-\left|\begin{array}{ccc}
\tilde{x}_{B 1}^{*} & \tilde{y}_{B 1}^{*} & \tilde{z}_{B 1}^{*} \\
\tilde{x}_{B 3}^{*} & \tilde{y}_{B 3}^{*} & \tilde{z}_{B 3}^{*} \\
\tilde{x}_{B 4}^{*} & \tilde{y}_{B 4}^{*} & \tilde{z}_{B 4}^{*}
\end{array}\right|=0,
$$

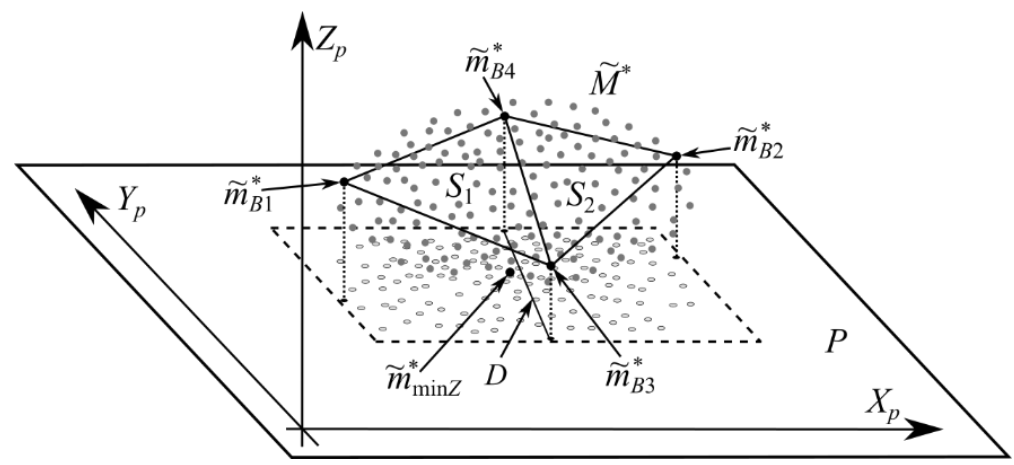

Fig. 5. Splitting of cloud of points on visible and hidden parts.

$$
S_{2}\left(\tilde{x}^{*}, \tilde{y}^{*}, \tilde{z}^{*}\right)=\left|\begin{array}{lll}
\tilde{y}_{B 2}^{*} & \tilde{z}_{B 2}^{*} & 1 \\
\tilde{y}_{B 3}^{*} & \tilde{z}_{B 3}^{*} & 1 \\
\tilde{y}_{B 4}^{*} & \tilde{z}_{B 4}^{*} & 1
\end{array}\right| \tilde{x}^{*}+\left|\begin{array}{lll}
\tilde{z}_{B 2}^{*} & \tilde{x}_{B 2}^{*} & 1 \\
\tilde{z}_{B 3}^{*} & \tilde{x}_{B 3}^{*} & 1 \\
\tilde{z}_{B 4}^{*} & \tilde{x}_{B 4}^{*} & 1
\end{array}\right| \tilde{y}^{*}+\left|\begin{array}{lll}
\tilde{x}_{B 2}^{*} & \tilde{y}_{B 2}^{*} & 1 \\
\tilde{x}_{B 3}^{*} & \tilde{y}_{B 3}^{*} & 1 \\
\tilde{x}_{B 4}^{*} & \tilde{y}_{B 4}^{*} & 1
\end{array}\right| \tilde{z}^{*}-\left|\begin{array}{ccc}
\tilde{x}_{B 2}^{*} & \tilde{y}_{B 2}^{*} & \tilde{z}_{B 2}^{*} \\
\tilde{x}_{B 3}^{*} & \tilde{y}_{B 3}^{*} & \tilde{z}_{B 3}^{*} \\
\tilde{x}_{B 4}^{*} & \tilde{y}_{B 4}^{*} & \tilde{z}_{B 4}^{*}
\end{array}\right|=0 .
$$

2) If the maximum value of coordinate $\tilde{z}_{B j}^{*}$ corresponds to points $\tilde{m}_{B 3}^{*}$ or $\tilde{m}_{B 4}^{*}$, then planes $S_{1}$ and $S_{2}$ are defined according to equations: 


$S_{1}\left(\tilde{x}^{*}, \tilde{y}^{*}, \tilde{z}^{*}\right)=\left|\begin{array}{lll}\tilde{y}_{B 1}^{*} & \tilde{z}_{B 1}^{*} & 1 \\ \tilde{y}_{B 2}^{*} & \tilde{z}_{B 2}^{*} & 1 \\ \tilde{y}_{B 4}^{*} & \tilde{z}_{B 4}^{*} & 1\end{array}\right| \tilde{x}^{*}+\left|\begin{array}{lll}\tilde{z}_{B 1}^{*} & \tilde{x}_{B 1}^{*} & 1 \\ \tilde{z}_{B 2}^{*} & \tilde{x}_{B 2}^{*} & 1 \\ \tilde{z}_{B 4}^{*} & \tilde{x}_{B 4}^{*} & 1\end{array}\right| \tilde{y}^{*}+\left|\begin{array}{lll}\tilde{x}_{B 1}^{*} & \tilde{y}_{B 1}^{*} & 1 \\ \tilde{x}_{B 2}^{*} & \tilde{y}_{B 2}^{*} & 1 \\ \tilde{x}_{B 4}^{*} & \tilde{y}_{B 4}^{*} & 1\end{array}\right| \tilde{z}^{*}-\left|\begin{array}{lll}\tilde{x}_{B 1}^{*} & \tilde{y}_{B 1}^{*} & \tilde{z}_{B 1}^{*} \\ \tilde{x}_{B 2}^{*} & \tilde{y}_{B 2}^{*} & \tilde{z}_{B 2}^{*} \\ \tilde{x}_{B 4}^{*} & \tilde{y}_{B 4}^{*} & \tilde{z}_{B 4}^{*}\end{array}\right|=0$,
$S_{2}\left(\tilde{x}^{*}, \tilde{y}^{*}, \tilde{z}^{*}\right)=\left|\begin{array}{lll}\tilde{y}_{B 2}^{*} & \tilde{z}_{B 2}^{*} & 1 \\ \tilde{y}_{B 1}^{*} & \tilde{z}_{B 1}^{*} & 1 \\ \tilde{y}_{B 4}^{*} & \tilde{z}_{B 4}^{*} & 1\end{array}\right| \tilde{x}^{*}+\left|\begin{array}{lll}\tilde{z}_{B 2}^{*} & \tilde{x}_{B 2}^{*} & 1 \\ \tilde{z}_{B 1}^{*} & \tilde{x}_{B 1}^{*} & 1 \\ \tilde{z}_{B 4}^{*} & \tilde{x}_{B 4}^{*} & 1\end{array}\right| \tilde{y}^{*}+\left|\begin{array}{lll}\tilde{x}_{B 2}^{*} & \tilde{y}_{B 2}^{*} & 1 \\ \tilde{x}_{B 1}^{*} & \tilde{y}_{B 1}^{*} & 1 \\ \tilde{x}_{B 4}^{*} & \tilde{y}_{B 4}^{*} & 1\end{array}\right| \tilde{z}^{*}-\left|\begin{array}{lll}\tilde{x}_{B 2}^{*} & \tilde{y}_{B 2}^{*} & \tilde{z}_{B 2}^{*} \\ \tilde{x}_{B 1}^{*} & \tilde{y}_{B 1}^{*} & \tilde{z}_{B 1}^{*} \\ \tilde{x}_{B 4}^{*} & \tilde{y}_{B 4}^{*} & \tilde{z}_{B 4}^{*}\end{array}\right|=0$.

After defining of planes $S_{1}$ and $S_{2}$, which separate visible and hidden parts it is necessary to define the straight line $D$ on the plane $P$, which will divide projections of points of $\tilde{M}^{*}$ in two parts corresponding to $S_{1}$ and $S_{2}$. For the first case the line $D$ equation will be defined as follows:

$$
D\left(\tilde{x}^{*}, \tilde{y}^{*}\right)=\left(\tilde{y}^{*}-\tilde{y}_{B 3}^{*}\right)\left(\tilde{x}_{B 3}^{*}-\tilde{x}_{B 4}^{*}\right)+\left(\tilde{x}^{*}-\tilde{x}_{B 3}^{*}\right)\left(\tilde{y}_{B 4}^{*}-\tilde{y}_{B 3}^{*}\right)=0
$$

and for the second:

$$
D\left(\tilde{x}^{*}, \tilde{y}^{*}\right)=\left(\tilde{y}^{*}-\tilde{y}_{B 1}^{*}\right)\left(\tilde{x}_{B 1}^{*}-\tilde{x}_{B 2}^{*}\right)+\left(\tilde{x}^{*}-\tilde{x}_{B 1}^{*}\right)\left(\tilde{y}_{B 2}^{*}-\tilde{y}_{B 1}^{*}\right)=0
$$

Then from cloud of points $\tilde{M}^{*}$ is selected cloud of points $\tilde{M}_{P}$, containing only visible part of points of projection to the plane $P$. Belonging of point $\tilde{m}_{i}^{*}$ to the cloud $\tilde{M}_{P}$ defining by help of the following conditions:

$$
\tilde{m}_{i}^{*} \in \tilde{M}_{P}, \text { if }\left(D\left(\tilde{m}_{i}^{*}\right)>0 \text { and } S_{1}\left(\tilde{m}_{i}^{*}\right)<0\right) \text { or }\left(D\left(\tilde{m}_{i}^{*}\right) \leq 0 \text { and } S_{2}\left(\tilde{m}_{i}^{*}\right)<0\right)
$$

The expression (9) shows that only those points from the cloud $\tilde{M}^{*}$, which are located left than straight line $D$ and lower than plane $S_{1}$ or right than line $D$ and lower than plane $S_{2}$ will be included to cloud $\tilde{M}_{P}$. Next the projection of $\tilde{M}_{P}$ to plane $P$ is divided in rectangle parts with the set step $\Delta x$ and $\Delta y$ along axis $x$ and $y$ accordingly and for each part density $\rho$ is calculated:

$$
\rho_{i}=\frac{L_{i}}{\Delta x \Delta y}
$$

where $L_{i}$ is number of points belonging to $i$-th part. If $\rho_{i} \geq \rho^{*}$ then considering part has enough density of points for the next processing. At the same time to the poor quality of the model does not indicate the existence of separate areas with a low density of points, but the presence of areas of these regions, covering an area larger than the allowable. After finding such domain, it is necessary to repeat the scan in this area, but with modified parameters (VS orientation, turning the light, etc.).

8) After receiving the workpiece model that meets the requirements, it is saved in the proper format and used later to generate trajectories for TCP of SIR.

Below we will examine the quality of a generalized algorithm for creating models of workpieces for SIR using VS and evaluating the quality of the resulting CAD models.

\section{The study of the algorithm for determining the quality of creation of three-dimensional models}

In the described approach to obtaining a three-dimensional model of workpieces is important to determine the gaps in this model. To determine the performance of this algorithm the research was conducted on a model example. As initial data was used the cloud of points (fig. 6a) generated from CAD-model of detail (fig. 6b) and contains areas with lower than other regions density of points.

During the studying the initial cloud of points was transformed to cloud $\tilde{M}^{*}$ according to (1) and (3). The matrix $R_{p}^{*}$ from (1) set the rotation relative to the $x$-axis of base CS on angles from 90 to -90 degrees with step 60 degrees, and on angle 15 degrees relative to z-axis of CS $X_{p} Y_{p} Z_{p}$ (see fig. 7b-10b). After that according to (4)-(9) invisible part of points were deleted. The resulting projection on the plane was divided into plots of $3 \times 3 \mathrm{~mm}$, for which by means of (10) the density of points was calculated.

The results of these calculations are shown on figures 7-10. On this figures: (a) is location of projection plane $P$ relative to the initial model; (b) is projection of cloud of points to the plane $P$; (c) is projection of cloud of points $\tilde{M}^{*}$ to the plane $P$; (d) is density map (the closer color to red the higher the density of points); (e) is map of gaps (blue indicates the areas where the density of the points below the minimum 1 point $/ \mathrm{mm}^{2}$ ). 
Figures show that using of (4)-(9) at certain angles allow to determine the presence of gaps in the original cloud of points (see. fig. 7b-c and 9b-c). Analysis the resulting density maps showing that on them are presented not only areas of reduced but with increased points density. The presence of areas of higher points density indicates that considering part is rotated towards the plane of projection on angle close to 90 degree and to estimate the quality of the model on this part the plane of projection should be additionally rotated.

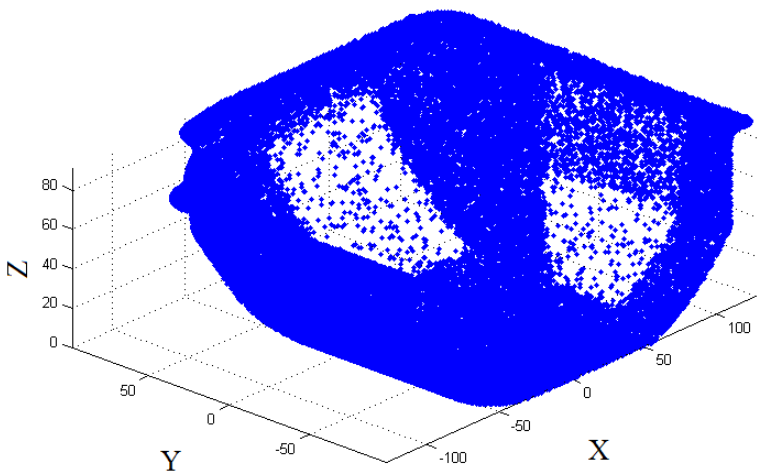

(a) cloud of points for model example

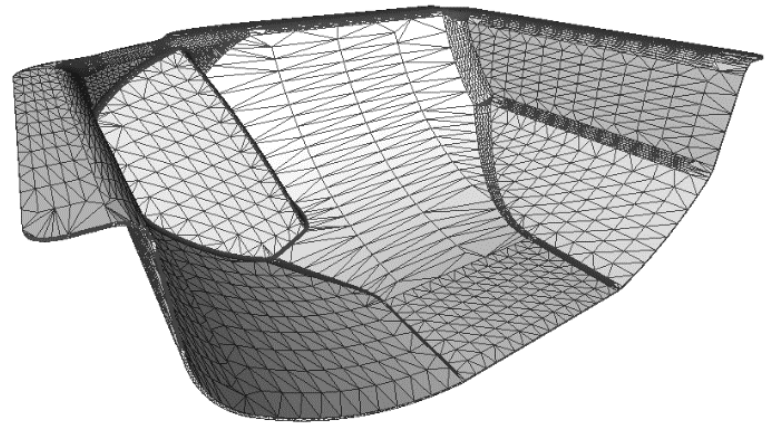

(b) CAD-model of workpiece

Fig. 6. The research results of the algorithm for determining the quality of creation of three-dimensional models
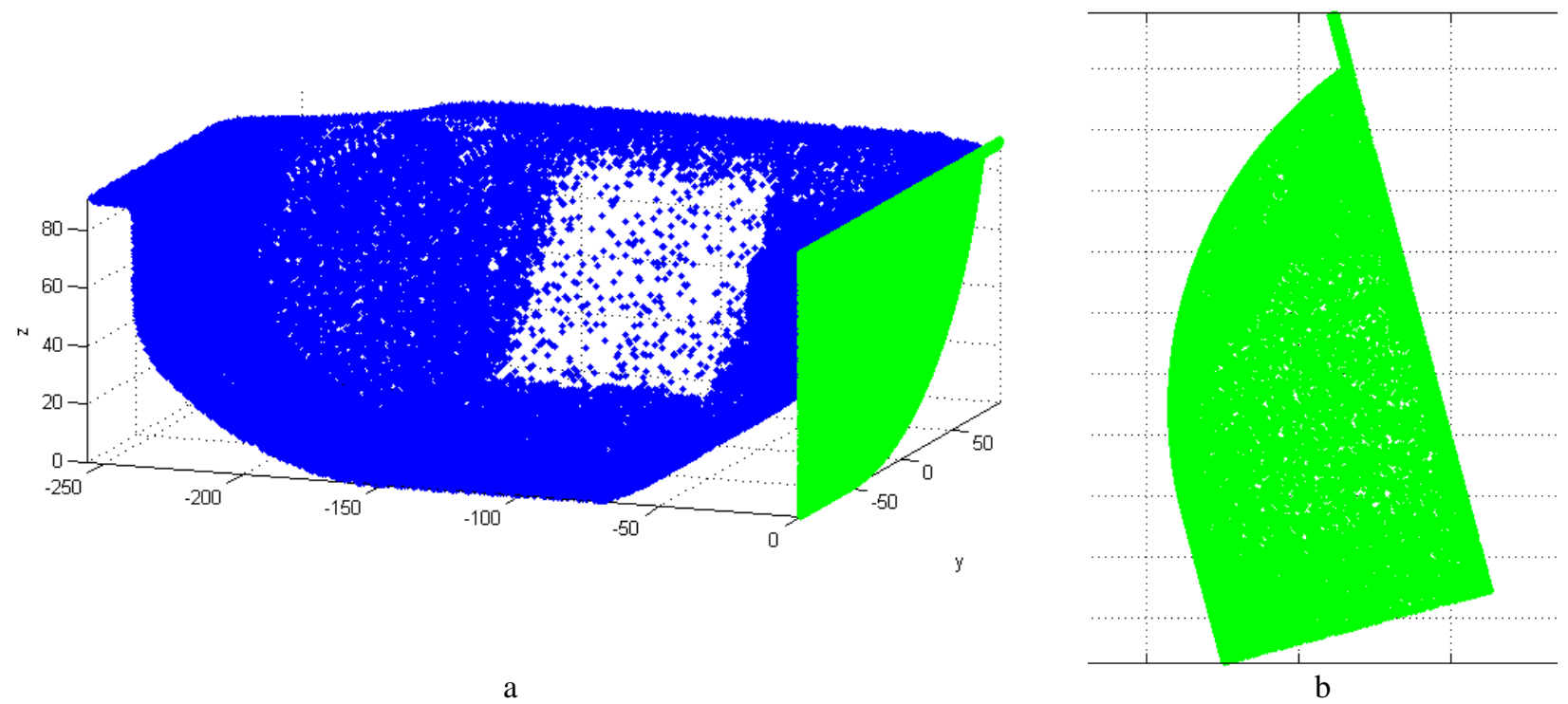



c

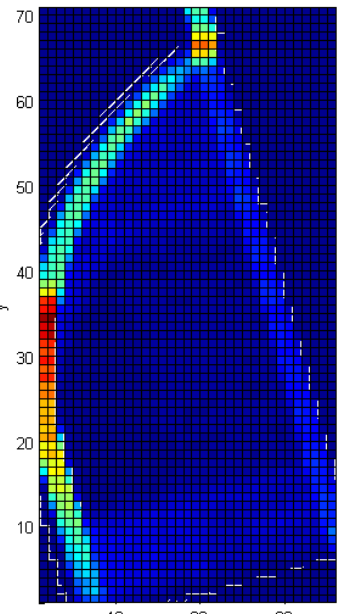

d

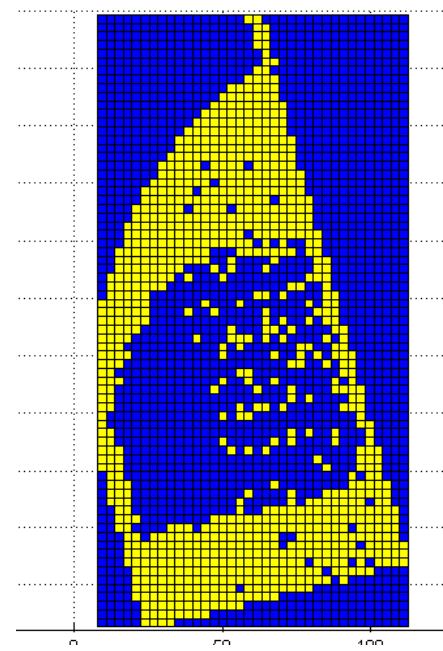

e

Fig. 7. Results of calculation of points density under rotation of projection plane on $90^{\circ}$ 


\section{TH DAAAM INTERNATIONAL SYMPOSIUM ON INTELLIGENT MANUFACTURING AND AUTOMATION}
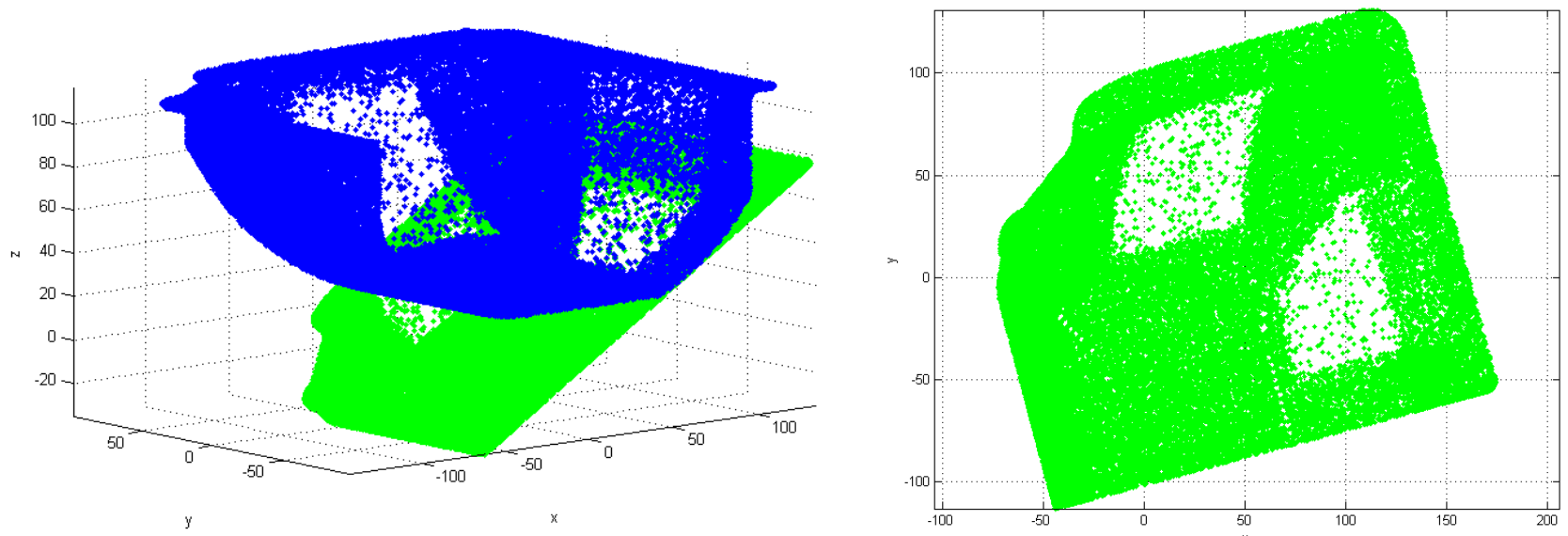

a
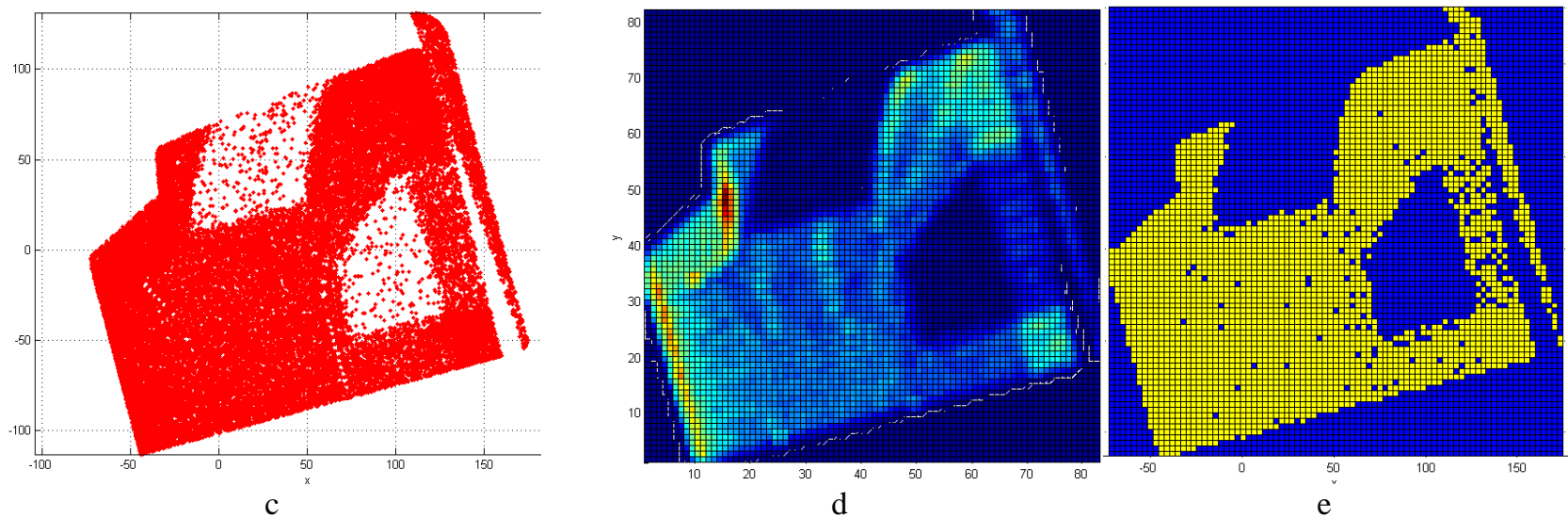

Fig. 8. Results of calculation of points density under rotation of projection plane on $30^{\circ}$
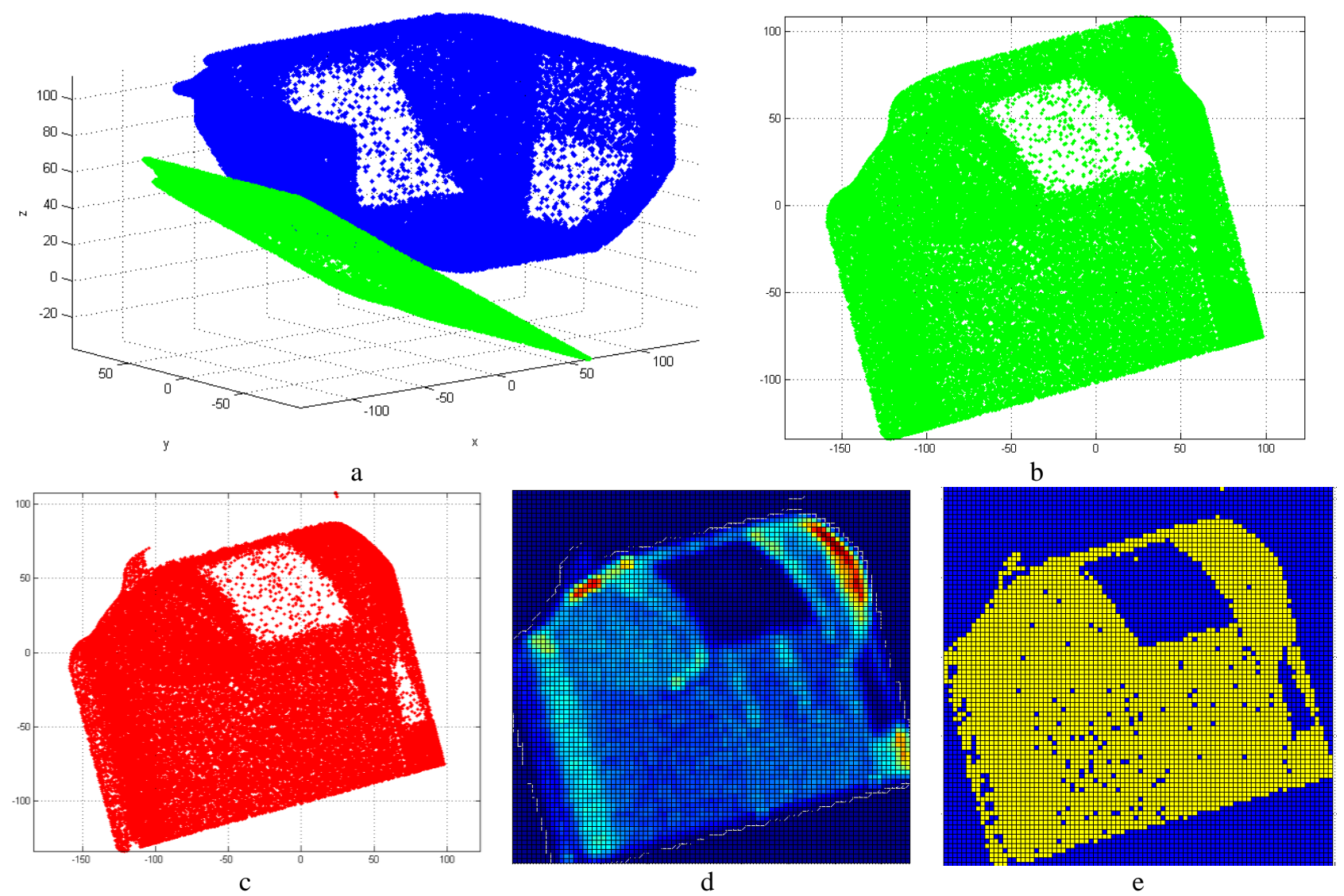

Fig. 9. Results of calculation of points density under rotation of projection plane on $-30^{\circ}$ 

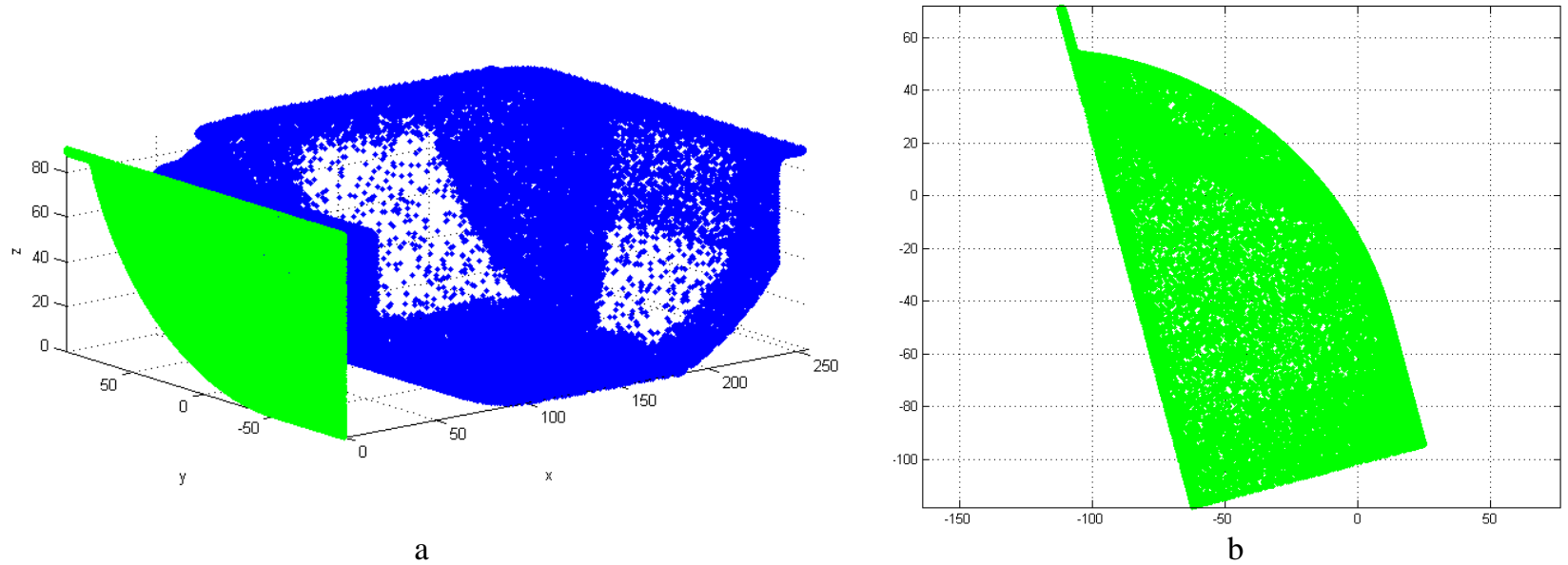

a

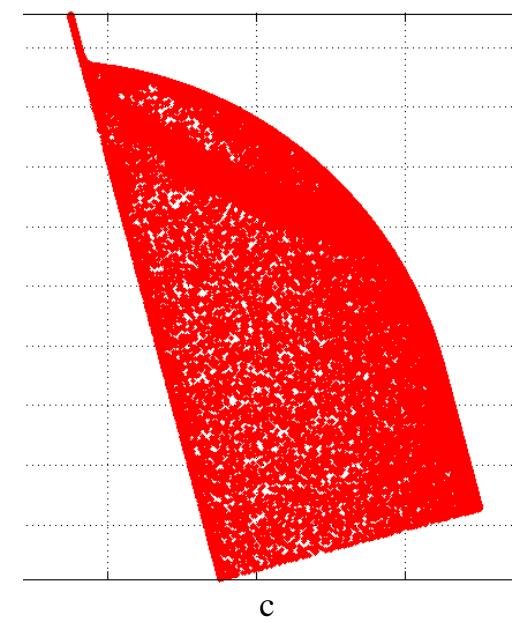

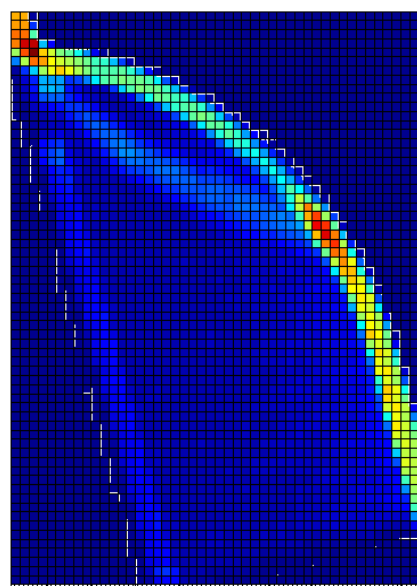

d

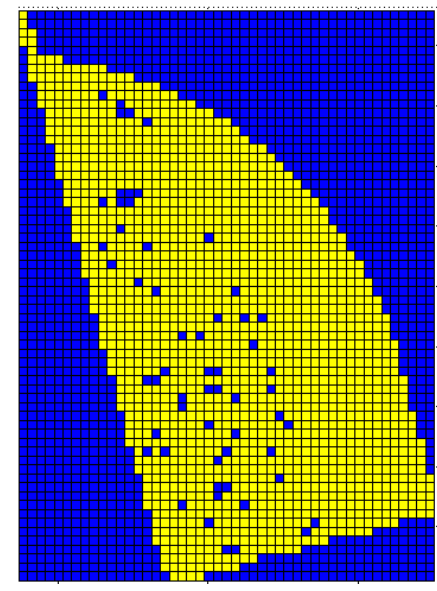

e

Fig. 10. Results of calculation of points density under rotation of projection plane on $-90^{\circ}$

It should be noted that when using the proposed algorithm it is possible to leave the analysis of threedimensional point clouds of high density and go to the analysis of the small resolution raster images. That requires ten times less processing power than using traditional algorithms of matching of three-dimensional images. In particular, if the workpiece has a technological holes, recesses, etc., then obtained density maps will correspond to areas of low point density. In this case, the approximate orientation of the workpiece can be calculated on the basis of the analysis of detected areas of low density, using standard and well developed algorithms for the analysis of raster images.

\section{Conclusion}

In the paper algorithm of determine the gaps in the point clouds obtained after scanning of the workpieces with the help of vision systems is proposes. The analysis of quality of the obtained cloud is offered to perform by means of relatively simple, well known and tested algorithms for processing raster images. The efficiency and effectiveness of this algorithm is confirmed on a model example.

\section{Acknowledgment}

This work is partially supported by the Ministry of Education and Science of Russian Federation (Contract \#14.604.21.0054 of 30.06.2014, RFMEFI60414X0054).

\section{References}

[1] Berthold K.P. Horn, Robot Vision, MIT Press, 480 p.

[2] D. Forsyth, J. Ponce, Computer Vision: A Modern Approach, Pearson Education, 2012, 761 p.

[3] L. G. Shapiro, G. C. Stockman, Computer Vision, Prentice Hall, 2001, 580 p.

[4] R. C. Gonzalez, R. E. Woods, Digital Image Processing, Pearson Education, 2011, 976 p.

[5] E. Trucco, A. Verri, Introductory Techniques for 3-D Computer Vision, Prentice Hall, 1998.

[6] Pugh A., Robot Vision, Springer-Verlag, 1983, 320 p. 
[7] Filaretov V., Zuev A., Yukhimets D., Gubankov A., Mursalimov E., The Automatization Method of Processing of Flexible Parts without their Rigid Fixation, Procedia Engineering, 100 (2015) 4-13.

[8] Filaretov V., Yukhimets D., Zuev A., Gubankov A., Mursalimov E., An new approach for automatization of cutting of flexible items by using multilink manipulators with vision system, Proc. of 22nd International Symposium on Power Electronics, Electrical Drives, Automation and Motion (SPEEDAM2014), Ischia Italy. (2014) 1330-1335.

[9] Korn G. A., Korn T. M., Mathematical Handbook for Scientists and Engineers, 2nd. ed., McGraw-Hill, N. Y., $1968,720 \mathrm{p}$. 